\title{
Genotypic differences of MCAD deficiency in the Asian population: Novel genotype and clinical symptoms preceding newborn screening notification
}

Regina Ensenauer, $M D^{1,2}$, Jennifer L. Winters, Patricia A. Parton, $M D^{4}$, David F. Kronn, $M D^{4}$, Jong-Won Kim, $M D, P h D^{5}$, Dietrich Matern, $M D^{1-3}$, Piero Rinaldo, $M D, P h D^{1-3}$, and Si Houn Hahn, $M D, P h D^{1-3}$

\begin{abstract}
Purpose: In contrast to its high prevalence in Caucasians, medium-chain acyl-CoA dehydrogenase (MCAD) deficiency is reported to be an extremely rare metabolic disorder in the Asian population. The common MCAD gene (ACADM) mutation 985A $>$ G (p.K329E), accounting for the majority of cases in Caucasians, has not been detected in this ethnic group, and the spectrum of $A C A D M$ mutations has remained unknown. Method: Biochemical genetic testing including plasma acylcarnitine and urine acylglycine analyses, as well as sequencing of ACADM was performed in a Korean family with a newborn who had an elevated octanoyl (C8) carnitine concentration by newborn screening (NBS). Genotyping of 50 Korean newborns with normal NBS results was performed. Result: We report the identification of the first Korean patient with MCAD deficiency, caused by a novel missense mutation in ACADM, 843A $>T$ (R281S), and a 4-bp deletion, c.449_452delCTGA. The patient became symptomatic before notification of the abnormal NBS result. Both the father and a brother who were identified as carriers for the 4-bp deletion had mildly elevated plasma $\mathrm{C} 8$ and $\mathrm{C} 10: 1$ carnitine concentrations, whereas the acylcarnitine profile was normal in the mother who carries the missense mutation. Conclusion: The 4-bp deletion may represent a common Asian ACADM mutation, considering that it recently has also been found in two of the three Japanese patients in whom genotyping was performed. Greater availability of MCAD mutation analysis is likely to unravel the molecular basis of MCAD deficiency in the Asian population that might differ from Caucasians. Genet Med 2005:7(5):339-343.
\end{abstract}

Key Words: MCAD deficiency, Korean, Asian, genotype, newborn screening

Since its first report more than 25 years ago, medium-chain acyl-CoA dehydrogenase (MCAD; EC 1.3.99.3) deficiency (MIM 201450) has been recognized as the most commonly diagnosed fatty acid oxidation disorder. ${ }^{1,2}$ Newborn screening (NBS) based on tandem mass spectrometry (MS/MS) and confirmatory mutation analysis detected a frequency for MCAD deficiency of 1 in 8,400 in Bavaria (Southern Germany) to 1 in 15,000 in the United States. ${ }^{3,4}$ However, the disorder is extremely rare among individuals of Asian background. The frequency of MCAD deficiency in Japan was estimated to be only 1 in 1,061,000 (based on screening for the common mutation $985 \mathrm{~A}>\mathrm{G})^{5}$ before a recent pilot study for expanded NBS, which led to the identification of the first two affected Japanese

\footnotetext{
From the ${ }^{1}$ Biochemical Genetics Laboratory, Departments of Laboratory Medicine and Pathology, ${ }^{2}$ Medical Genetics, and ${ }^{3}$ Pediatric and Adolescent Medicine, Mayo Clinic College of Medicine, Rochester, Minnesota; ${ }^{4}$ Department of Pediatrics, New York Medical College, New York; and ${ }^{5}$ Department of Laboratory Medicine, Samsung Medical Center, Sung Kyun Kwan University, School of Medicine, Seoul, Korea.

Sihoun Hahn, MD, PhD, Biochemical Genetics Laboratory, Department of Laboratory Medicine and Pathology, Mayo Clinic College of Medicine, 200 First Street SW, Rochester, MN 55905.

Received: January 3, 2005.

Accepted: February 25, 2005.
}

DOI: 10.1097/01.GIM.0000164548.54482.9D individuals among 102,200 screened newborns. ${ }^{6}$ The precise genotype of these two patients, however, has not been reported. Very recently, 3 of 88,886 newborns in the Hiroshima area were diagnosed with MCAD deficiency, two of whom carried a novel 4-bp deletion, c.449_452delCTGA, in a compound heterozygous form. ${ }^{7}$

The gene for MCAD, ACADM, is located on chromosome $1 \mathrm{p} 31$ and consists of 12 exons spanning $44 \mathrm{~kb}$ of DNA. ${ }^{8}$ The $985 \mathrm{~A}>\mathrm{G}$ (p.K329E) mutation is the most common gene variant accounting for $85 \%$ to $90 \%$ of alleles detected in clinically symptomatic Caucasian patients of Northern European descent but only for $60 \%$ of defective alleles of patients diagnosed by NBS. ${ }^{3,9,10}$. In addition, approximately 40 other, mostly private mutations have been reported. ${ }^{3,4,11}$ The majority of the disease-causing ACADM mutations identified so far are missense mutations (21 entries in The Human Gene Mutation Database, Cardiff ${ }^{12}$ ), but several deletion mutations, insertions, and splice-site mutations have been described as well. ${ }^{3,4,11,13,14}$ Estimates for the frequency of homozygosity for the common $985 \mathrm{~A}>\mathrm{G}$ mutation among Northern Europeans range from 1 in 6,400 to 1 in 46,000 with a carrier frequency of $1 \%$ to $2 \% .4,15$ This mutation, presumably a founder effect from a single person in a Germanic tribe, is very rare in other populations including Asian, African American, Southern Euro- 
pean, and North African. ${ }^{5}$ Population-based studies have not identified any mutation carriers among more than 800 healthy Japanese newborns. ${ }^{15,16}$ Here, we report the identification of the first Korean patient with MCAD deficiency, bearing a novel genotype and presenting in the first week of life.

\section{MATERIALS AND METHODS}

\section{Case report}

The male infant is the third child born in the state of New York to nonconsanguineous healthy Korean parents following a normal pregnancy and spontaneous term delivery. The 30year-old mother, G7/P3043, had three previous miscarriages and one ectopic pregnancy. The two older brothers, ages 2 and 4 years, have been healthy. The patient's APGAR scores were normal, and birth weight, length, and head circumference were unremarkable. A blood sample for NBS was collected at his 3rd day of life directly before discharge home. Because of poor feeding, he was presented to the pediatrician on his 5th day of life and promptly hospitalized due to dehydration and acidosis. Tremor was noted, and blood glucose was measured at 40 $\mathrm{mg} / \mathrm{dL}$. Intravenous glucose was continued until the 8 th day of life. After hospital discharge on that same day, the family was notified several hours later by the state NBS laboratory of a positive screening result with an octanoyl (C8) carnitine concentration of $36.32 \mu \mathrm{mol} / \mathrm{L}$ (cut-off $<0.3$ ) suggestive of MCAD deficiency.

After the diagnosis was confirmed, treatment was initiated consisting of carnitine supplementation $(50 \mathrm{mg} / \mathrm{kg}$ per day) and avoidance of fasting. Breastfeeding was continued. The patient has remained healthy and developed appropriately in the first 5 months of life.

\section{Biochemical and molecular genetic analyses}

Confirmatory diagnostic testing in the patient as well as his parents and two siblings included plasma acylcarnitine, urine acylglycine, and molecular genetic analyses. Biochemical genetic assays were performed using established procedures. ${ }^{17}$ With parental consent and approval by Mayo Clinic's Institutional Review Board (IRB), genomic DNA was extracted from whole blood of the patient and family members and subjected to mutation analysis. All 12 exons including adjacent splice sites of ACADM (GenBank accession no. AH002873) were amplified by PCR using intron-located primers. Mutation analysis was performed by direct sequencing of PCR-amplified fragments using an ABI Prism 3100 DNA Analyzer (96-capillary; Perkin-Elmer Applied Biosystems, Foster City, CA). Primer sequences are available upon request. In addition, with approval of Mayo Clinic's IRB, DNA was extracted from 50 randomly selected and anonymized Korean NBS cards from Samsung Medical Center, Seoul (Korea), and genotyped by sequencing exons 6 and 9.

\section{RESULTS}

\section{Biochemical genetic analyses}

The patient's follow-up plasma acylcarnitine profile was characteristic of MCAD deficiency (Fig. 1a). Urine hexanoylglycine and suberylglycine concentrations were elevated at 36.4 $\mu \mathrm{g} / \mathrm{mg}$ creatinine (reference range $0.2-1.9$ ) and $32.9 \mu \mathrm{g} / \mathrm{mg}$ creatinine (reference range $0.01-11.0$ ), respectively. Total and free plasma carnitine concentrations were low at $11 \mu \mathrm{mol} / \mathrm{L}$ (reference range 19-59) and $7 \mu \mathrm{mol} / \mathrm{L}$ (reference range 1246), respectively. CPK and ammonia levels were normal; however, liver transaminases were mildly elevated (SGOT $55 \mathrm{U} / \mathrm{L}$, normal range 4-35; SGPT $66 \mathrm{U} / \mathrm{L}$, normal range 2-40).

The plasma acylcarnitine profile of the 4-year-old brother showed mild elevations of $\mathrm{C} 8$ and decenoyl (C10:1) carnitine concentrations; decanoyl (C10) carnitine concentration was in the upper normal range (Table 1; Fig. 1b). Similarly, the father's plasma acylcarnitine profile revealed an elevated concentration of $\mathrm{C} 10: 1$; the $\mathrm{C} 8$ and $\mathrm{C} 10$ carnitine concentrations were in the upper normal range (Table 1; Fig. 1c). Both the 2 -year-old brother and the mother had normal acylcarnitine profiles (Table 1; Fig. 1d). Urine acylglycine analyses of all four relatives were entirely normal.

\section{Molecular genetic analysis}

Mutation analysis of the patient's DNA revealed compound heterozygosity for a novel missense mutation and a 4-bp deletion in ACADM (Fig. 2). The mutant allele originating from the father was the 4-bp deletion in exon 6 (c.449_452delCTGA) causing a frameshift and premature stop codon, which is expected to result in a truncated protein. The other mutant allele inherited from the mother was a missense mutation in exon $9(843 \mathrm{~A}>\mathrm{T})$, resulting in substitution of an arginine by a serine at position 281 of the precursor protein. Arginine at position 281 is conserved across species. The 4 -year-old brother was heterozygous for c.449_452delCTGA, the 2-year-old brother did not carry any of the mutations (Table 1). None of the mutations was detected in any of the 100 alleles of the randomly selected Korean newborns with normal NBS test results.

\section{DISCUSSION}

Although considered the most common fatty acid oxidation disorder, MCAD deficiency is a rare metabolic condition in Asians, $, 5,15,16$ and only limited information is available from the few published case reports. A 6-week-old male Thai infant who died after his second metabolic decompensation was presumed to have MCAD deficiency based on the finding of mild to moderate dicarboxylic aciduria, moderate 2-hydroxy glutaric aciduria, and massive lactic aciduria. ${ }^{18}$ Another suspected case of MCAD deficiency was reported from China, with dicarboxylic aciduria and the presence of trace hexanoylglycine in urine. ${ }^{19}$ Both cases were not confirmed by plasma acylcarnitine or molecular genetic analyses. 

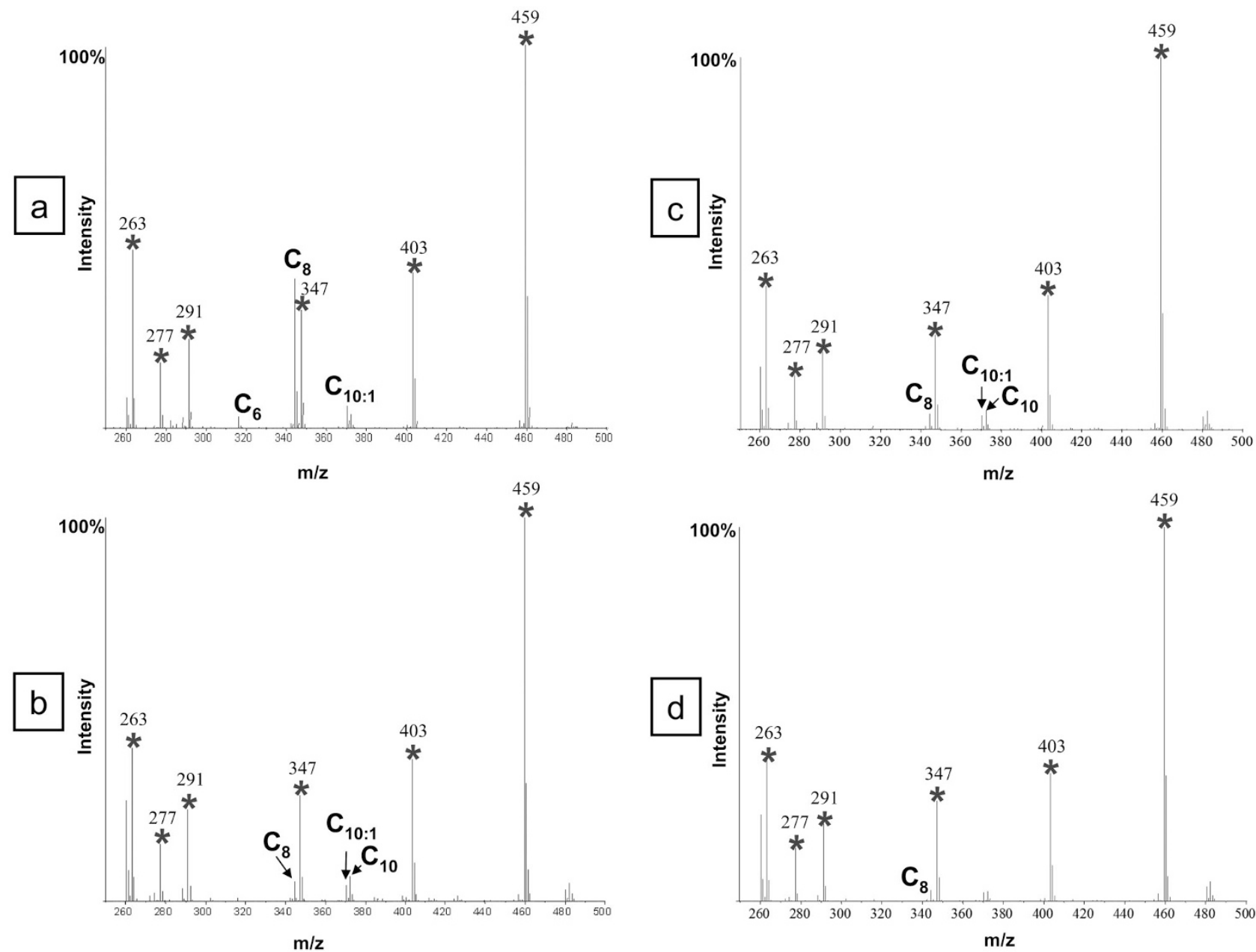

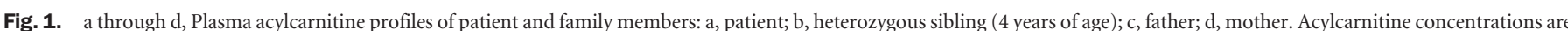

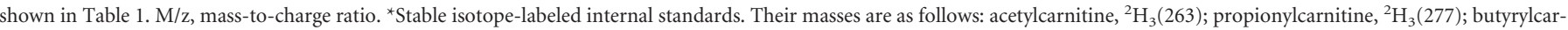
nitine, ${ }^{2} \mathrm{H}_{3}(291)$; octanoylcarnitine, ${ }^{2} \mathrm{H}_{3}$ (347); dodecanoylcarnitine, ${ }^{2} \mathrm{H}_{3}(403)$; and palmitoylcarnitine, ${ }^{2} \mathrm{H}_{3}(459)$.

Table 1

Genotype and plasma acylcarnitine concentrations $(\mu \mathrm{mol} / \mathrm{L})$ of the patient and his first-degree relatives

\begin{tabular}{|c|c|c|c|c|c|c|c|}
\hline \multirow[b]{2}{*}{ Subject } & \multicolumn{2}{|c|}{ Genotype } & \multicolumn{5}{|c|}{ Biochemical phenotype } \\
\hline & Allele 1 & Allele 2 & $\begin{array}{l}\text { Hexanoyl } \\
\text { (C6) }\end{array}$ & $\begin{array}{l}\text { Octanoyl } \\
\text { (C8) }\end{array}$ & $\begin{array}{c}\text { Decanoyl } \\
\text { (C10) }\end{array}$ & $\begin{array}{c}\text { Decenoyl } \\
\text { (C10:1) }\end{array}$ & $\mathrm{C} 8 / \mathrm{C} 10$ ratio \\
\hline Proband (newborn) & c.449_452delCTGA & $843 \mathrm{~A}>\mathrm{T}$ & 0.33 & 4.58 & 0.41 & 0.71 & 11.32 \\
\hline Sibling (2 y old) & Wild type & Wild type & 0.02 & 0.18 & 0.12 & 0.10 & 1.55 \\
\hline Sibling (4 y old) & c.449_452delCTGA & Wild type & 0.14 & 0.71 & 0.87 & 0.59 & 0.81 \\
\hline Mother & Wild type & $843 \mathrm{~A}>\mathrm{T}$ & 0.09 & 0.44 & 0.36 & 0.34 & 1.21 \\
\hline Father & c.449_452delCTGA & Wild type & 0.13 & 0.62 & 0.77 & 0.54 & 0.81 \\
\hline \multirow[t]{2}{*}{ Reference ranges } & Wild type & Wild type & Child $<0.22$ & Child $<0.44$ & Child $<0.91$ & Child $<0.45$ & Child $<4.36$ \\
\hline & & & Adult $<0.16$ & Adult $<0.77$ & Adult $<0.88$ & Adult $<0.46$ & Adult $<2.28$ \\
\hline
\end{tabular}

It was only in 2002, through a nationwide pilot program for NBS using MS/MS, when the first two Japanese newborns with MCAD deficiency were identified. ${ }^{6}$ A third Japanese patient was diagnosed after symptomatic hypoglycemia. ${ }^{6}$ Very re- cently, three additional patients with MCAD deficiency were detected in a Hiroshima NBS program. ${ }^{7}$ However, the spectrum of $A C A D M$ mutations in the Asian population has remained unknown. Population studies have been conducted for 


\section{A Exon 6 control}

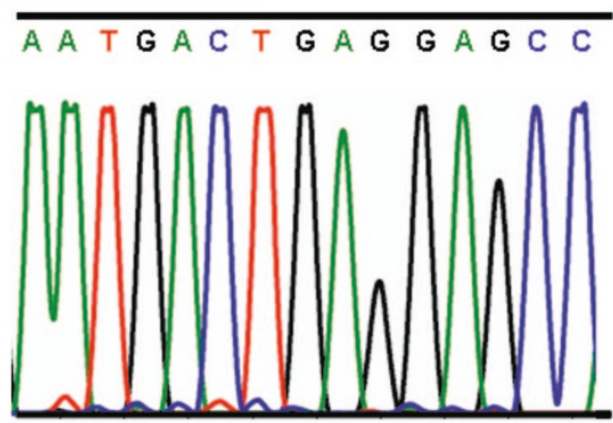

\section{c.449_452delCTGA}

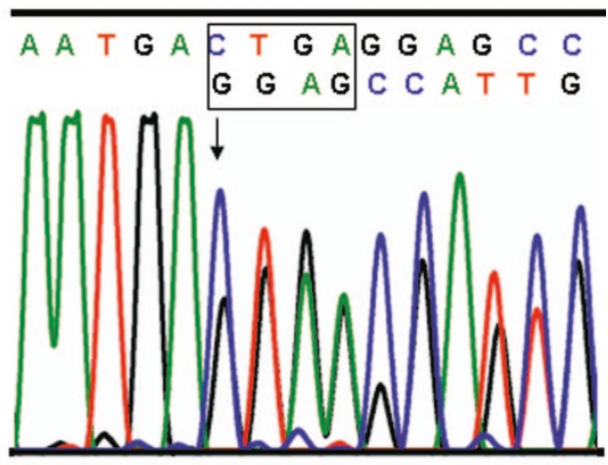

B Exon 9 control

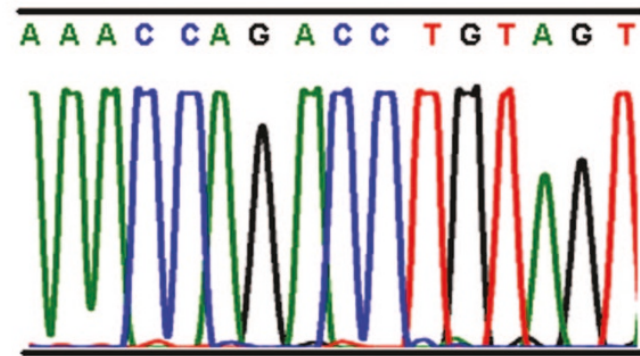

843A $>T(R 281 S)$

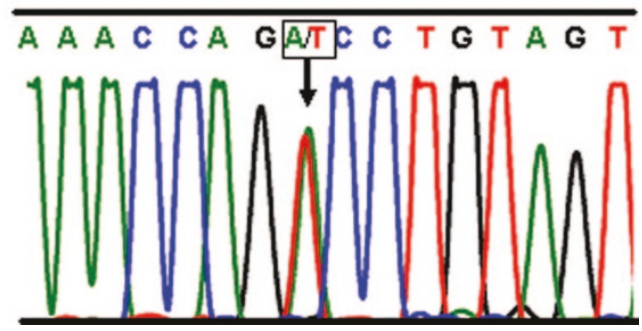

Fig. 2. Mutations identified in the patient by sequencing of $A C A D M$. a, Heterozygote for 4-bp deletion c.449_452delCTGA in exon 6 resulting in a frameshift. b, Heterozygote for $843 \mathrm{~A}>\mathrm{T}$ (p.R281S) in exon 9.

only the common $985 \mathrm{~A}>\mathrm{G}$ mutation and have shown that its carrier frequency is extremely low in Japan. ${ }^{15,16}$ Interestingly, the 4-bp deletion, c.449_452delCTGA, identified in the pre- sented Korean newborn was also detected in two of the three neonates from Hiroshima, ${ }^{7}$ and therefore, this deletion may represent a common Asian ACADM mutation; however, genotyping of additional Asian MCAD deficient patients is required. A cohort of Korean newborns did not reveal any deletion carriers; however, studies of the carrier frequency in other Asian populations, such as the Japanese, are still pending.

The present infant is the first patient of Korean descent identified with MCAD deficiency diagnosed by NBS and confirmed by positive biochemical and molecular genetic analyses. Of note, the patient's clinical course was remarkable for an episode of metabolic decompensation at the 5th day of life before notification of an abnormal NBS result. This case underscores the importance of rapid turnaround time in reporting and notification of abnormal results as an essential component of a NBS program.

Interestingly, the father and the 4-year-old brother of the patient who are carriers for the 4-bp deletion showed slight elevations of plasma C8 and/or C10:1 carnitines, whereas the mother who carries the novel missense mutation had an entirely normal acylcarnitine profile. This suggests that the degree of functional impact of a mutation on MCAD activity may be reflected on the metabolite level, and thus, some carriers of more deleterious mutations may show an abnormal plasma acylcarnitine profile. Heterozygosity for the $985 \mathrm{~A}>\mathrm{G}$ mutation was suggested to be associated with mildly elevated C8 carnitine concentrations in NBS blood spots. ${ }^{20}$ Future in vitro expression studies should focus on correlating effects of identified mutations at the protein level with the disease-specific metabolites. Of note, acylcarnitine profiles of individuals carrying one deleterious mutation may not be readily differentiated from profiles of individuals who are homozygous or compound heterozygous for mutations predicted to have less deleterious effects. However, the $\mathrm{C} 8 / \mathrm{C} 10$ ratio was helpful in the evaluation of this family, as seen for the deletion carriers reported in this study who showed significantly lower ratios (Table 1) than MCAD deficient patients with less deleterious mutations ("mild biochemical phenotype of MCAD deficiency"13). Greater availability of MCAD genotyping ${ }^{21}$ is likely to facilitate the evaluation of cases with milder metabolite profiles that are not typical but suggestive of a possible biochemical diagnosis of MCAD deficiency.

\section{References}

1. Gregersen N, Lauritzen R, Rasmussen K. Suberylglycine excretion in the urine from a patient with dicarboxylic aciduria. Clin Chim Acta 1976;70:417-425.

2. Matern D, Rinaldo P. Medium-chain acyl-coenzyme A dehydrogenase deficiency. In: GeneReviews: Genetic Disease Online Reviews at GeneTests-GeneClinics [database online: Initial posting: April 20, 2000; last update January 27, 2003]. University of Washington, Seattle. Available at: http://www.geneclinics.org Accessed January 2, 2005.

3. Maier EM, Liebl B, Röschinger W, Nennstiel-Ratzel U, Fingerhut R, Olgemöller B et al. Population spectrum of ACADM genotypes correlated to biochemical phenotypes in newborn screening for medium-chain acyl-CoA dehydrogenase deficiency. Hum Mutat 2005; 25:445-452 [Epub ahead of print].

4. Andresen BS, Dobrowolski SF, O’Reilly L, Muenzer J, McCandless SE, Frazier $\mathrm{DM}$ et al. Medium-chain acyl-CoA dehydrogenase (MCAD) mutations identified by MS/MS-based prospective screening of newborns differ from those observed in patients with clinical symptoms: Identification and characterization of 
a new, prevalent mutation that results in mild MCAD deficiency. Am J Hum Genet 2001;68:1408-1418.

5. Tanaka K, Gregersen N, Ribes A, Kim J, Kølvraa S, Winter V et al. A survey of the newborn populations in Belgium, Germany, Poland, Czech Republic, Hungary, Bulgaria, Spain, Turkey, and Japan for the G985 variant allele with haplotype analysis at the medium chain acyl-CoA dehydrogenase gene locus: Clinical and evolutionary consideration. Pediatr Res 1997;41:201-209.

6. Shigematsu Y, Hirano S, Hata I, Tanaka Y, Sudo M, Sakura N et al. Newborn mass screening and selective screening using electrospray tandem mass spectrometry in Japan. J Chromatogr B 2002;776:39-48.

7. Tajima G, Sakura N, Ono H, Kobayashi M, Hata I, Shigematsu Y. Medium-chain acyl-CoA dehydrogenase deficiency found through newborn screening by tandem mass spectrometry in Japan. J Inherit Metab Dis 2004;27(suppl):8.

8. Zhang ZF, Kelly DP, Kim JJ, Zhou Y, Ogden ML, Whelan AJ et al. Structural organization and regulatory regions of the human medium-chain acyl-CoA dehydrogenase gene. Biochemistry 1992;31:81-89.

9. Gregersen N, Blakemore AIF, Winter V, Andresen B, Kølvraa S, Bolund L et al. Specific diagnosis of medium-chain acyl-CoA dehydrogenase (MCAD) deficiency in dried blood spots by a polymerase chain reaction (PCR) assay detecting a point-mutation (G985) in the MCAD gene. Clin Chim Acta 1991; 203:23-34.

10. Yokota I, Coates PM, Hale DE, Rinaldo P, Tanaka K. Molecular survey of a prevalent mutation, 985A-to-G transition, and identification of five infrequent mutations in the medium-chain acyl-CoA dehydrogenase (MCAD) gene in 55 patients with MCAD deficiency. Am J Hum Genet 1991;49:1280-1291.

11. Andresen BS, Bross P, Udvari S, Kirk J, Gray G, Kmoch S et al. The molecular basis of medium-chain acyl-CoA dehydrogenase (MCAD) deficiency in compound het erozygous patients: is there correlation between genotype and phenotype? $\mathrm{Hum} \mathrm{Mol}$ Genet 1997;6:695-707.

12. The Human Gene Mutation Database, Cardiff. ACADM Nucleotide substitution (missense/nonsense). Available at: http://archive.uwcm.ac.uk/uwcm/mg/ns/1/ 118958.html. Accessed January 2, 2005.
13. Zschocke J, Schulze A, Lindner M, Fiesel S, Olgemoeller K, Hoffmann GF et al. Molecular and functional characterisation of mild MCAD deficiency. Hum Genet 2001;108:404-408

14. Korman SH, Gutman A, Brooks R, Sinnathamby T, Gregersen N, Andresen BS. Homozygosity for a severe novel medium-chain acyl-CoA dehydrogenase (MCAD) mutation IVS3$1 \mathrm{G}>\mathrm{C}$ that leads to introduction of a premature termination codon by complete missplicing of the MCAD mRNA and is associated with phenotypic diversity ranging from sudden neonatal death to asymptomatic status. Mol Genet Metab 2004;82:121-129.

15. Matsubara Y, Narisawa K, Tada K, Ikeda H, YeQi Y, Danks DM et al. Prevalence of p.K329E mutation in medium-chain acyl-CoA dehydrogenase gene determined from Guthrie cards. Lancet 1991;338:552-553.

16. Nagao M. Frequency of ${ }^{985}$ A-to-G mutation in medium-chain acyl-CoA dehydrogenase gene among patients with sudden infant death syndrome, Reye syndrome, severe motor and intellectual disabilities and healthy newborns in Japan. Acta Paediatr Jpn 1996;38:304-307.

17. Rinaldo $\mathrm{P}, \mathrm{Hahn} \mathrm{SH}, \mathrm{Matern} \mathrm{D}$. Inborn errors of amino acid, organic acid, and fatty acid metabolism. In: Burtis CA, Ashwood ER, Tietz NW, editors. Tietz Textbook of Clinical Chemistry, 4th ed., W.B. Saunders, 2005. In press.

18. Wasant P, Naylor E, Matsumoto I, Liammongkolkul S. Mitochondrial fatty acid oxidation disorders in Thai infants: a report of 3 cases. J Med Assoc Thai 2002; 85(suppl 2):S710-S719.

19. Pang CP, Law LK, Mak YT, Shek CC, Cheung KL, Mak TWL et al. Biochemica investigation of young hospitalized Chinese children: results over a 7-year period. Am J Med Genet 1997;72:417-421.

20. Lehotay DC, LePage J, Thomspon JR, Rockman-Greenberg C. Blood acylcarnitine levels in normal newborns and heterozygotes for medium-chain acyl-CoA dehydrogenase deficiency: a relationship between genotype and biochemical phenotype? J Inherit Metab Dis 2004;27:81-88.

21. McKinney JT, Longo N, Hahn SH, Matern D, Rinaldo P, Strauss AW et al. Rapid, comprehensive screening of the human medium chain acyl-CoA dehydrogenase gene. Mol Genet Metab 2004;82:112-120. 